\title{
THE CHALLENGE OF REDUCING THE RATE OF BLOOD CULTURE CONTAMINATION IN A GERIATRIC HOSPITAL IN ISRAEL
}

\author{
Leibovitz Arthur ${ }^{1}$, Zaidenstein Ronit ${ }^{2}$, Heymann Anthony ${ }^{3}$, Taichman Shoshana ${ }^{1}$, Mizrahi \\ Eliyahy $^{1}$, Molchanov Inna ${ }^{1}$, Lubart Emily ${ }^{1^{*}}$ \\ ${ }^{1}$ Shmuel Harofeh Geriatric Medical Center affiliated to Sackler School of Medicine, Tel-Aviv University, Israel. \\ ${ }^{2}$ Assaf Harofeh Medical Center, affiliated to Sackler School of Medicine, Tel-Aviv University \\ ${ }^{3}$ The Department of Family Medicine. The Sackler School of Medicine, Tel-Aviv University
}

\section{ABSTRACT}

This article describes our efforts to reduce the contamination rate in our geriatric hospital. Our program consisted of an educational stage and a second stage that screened the opinion of physicians of the geriatric hospital versus those of a general hospital with regard to blood culture sampling practices.

The blood culture contamination rate was reduced from $9 \%$ to $6 \%$ but this did not quite reach statistical significance $(p=0.21)$. The physicians' questionnaire results strengthen our perception that the difficulty in further reduction could be related to the uncooperative condition of many geriatric patients, thus requiring more hands in the process of blood drawing.

Our study indicates that such educational efforts should continue and emphasize that reduction in the contamination rate of blood samples is an important and achievable goal of the geriatric hospital system.

Keywords : blood stream infections, blood culture contamination, geriatric hospital.

\section{INTRODUCTION}

Blood stream infection (BSI) continues to be a major cause of morbidity and mortality in general hospitals and of increasing concern in geriatric hospitals $^{1,2}$. Blood cultures are important for the diagnosis and management of BSIs and it has become evident in recent years that contaminated blood cultures are common ${ }^{3,4}$, enormously costly ${ }^{5}$ and frequently confusing for clinicians ${ }^{6}$. Clinical studies of bloodstream infections have provided guidelines for differentiating true pathogens from contaminants or organisms of unknown significance ${ }^{7}$. However, a true "gold standard" for differentiating pathogens from contaminants does not exit ${ }^{4,8}$. Microorganisms such as Coagulasenegative Staphylococcus, Propionibacterium acnes, Micrococcus, Viridans-group streptococci, Corynebacterium or Bacillus species usually represent contaminations ${ }^{9,10}$, and rarely true bacteremia ${ }^{7}$. Despite numerous advances in blood culture methodology and systems in recent decades, an increasing proportion of blood culture isolates represents contaminations ${ }^{7}$. The ability of new systems and culture media to detect microorganisms, even in small numbers, may be

\section{*Corresponding author:}

Email: elubart@hotmail.com

http://dx.doi.org/10.20530/IJTA 31 10-13

ISSN 2320-138X (c) 2016 responsible in part for the observed increase in the proportion of blood cultures with contaminants ${ }^{3}$. Audit in our geriatric inpatient facility showed a high (around $10 \%$ ) proportion of blood culture contaminations before the year of 2009. General hospital departments, mainly emergency and neonatal units have reported efforts to reduce the contamination rates by using different strategies such as devoted teams, re-education programs and strict application of sterile methods, mostly with considerable success ${ }^{11-14}$. The range of contaminations they obtained was around 2 to $5 \%$ 11,12

Our literature search found no comparable studies in hospitals for geriatric patients so we cannot compare our rate with that of similar facilities.

Our high infection rate ${ }^{1,2}$ and the need to perform blood cultures and get accurate results in time, prompted us to establish a team dedicated to the study of the clinical aspects of taking blood cultures. This paper describes how our blood culture team (BCT) developed an interventional program with the aim to reduce the rate of blood culture contamination.

\section{METHODS}

The study was performed in a university affiliated 350-bed geriatric hospital with full onsite laboratories, including bacteriologic laboratory. Patients are admitted from the community, 
nursing-homes and general hospitals into acute, rehabilitation and long-term care wards.

The hospital's standard practice is to draw aseptically from a peripheral vein at least $20 \mathrm{ml}$ of blood. Equal portions of the blood samples are then inoculated into the sets of blood culture (aerobic and anaerobic). This procedure is performed by a physician, with the assistance of a nurse. The isolates considered as contaminants were: coagulase-negative Staphylococc species, Bacillus, Diphteroids, Corynebacterium, Micrococcus species, Viridans-group streptococci and Propionibacterium acnes.

The data were retrieved from the records of the bacteriological laboratory. The charts of the patients with blood cultures positive for microorganisms considered as contaminants were identified and examined. The contamination rate of the year of the education program was then compared with that of the following year.

The intervention was performed in two stages: the first stage physician education and the second stage a physician questionnaire. Each physician received personal letters with details of correct blood culture sampling techniques including the cleaning of venipuncture site, use of best antiseptic materials, changing to different needles in blood sampling and transfer to culture bottles.

In parallel, we organized discussions in small groups (3-5 physicians), followed by sessions of individual training on blood collection methods. An additional measure was to require the signature of the physician's name on each blood culture sample drawn.

Periodically (once in three months) reminders were sent to each physician with detailed explanation on blood culture collection techniques. All the physicians attended this program. It was started at the beginning of 2009 and completed within six months. The second stage consisted of a physicians' survey on the practical details of the blood sampling process including aspects of the interaction with the patients and the nurse aids. This survey was also undertaken in a group of physicians from different departments in a neighboring general hospital. The surveys were handed out during regular staff meetings in the two hospitals. The survey was based on a questionnaire prepared by the BCT together with colleagues from the general hospital. It included questions about the level of cooperation of the patients with the medical staff during the process of blood drawing, the level of the assistance provided by the nursing staff for the holding of and in the eventual patient's arm fixation. Items were graded on a Likert scale from 1 (lowest level) to 5 (highest level). Thirty residents from each hospital participated in the survey.

Institutional Review Board approval was obtained for this study.

\section{RESULTS}

The educational program was completed between January and June 2009. During 2009, 3142 blood cultures were drawn with 283 (9\%) of them found to be contaminated. In the following year, 2010, 3338 blood cultures were performed, and $206(6 \%)$ of them were contaminated. Although there was a decrease from $9 \%$ to $6 \%$ it was not statistically significant $(p=0.21)$.

The comparative analysis of the questionnaire from the geriatric hospital and the general hospital were available at the beginning of 2012 and revealed a significantly higher level of cooperation by the patients of the general hospital with the medical staff during the blood collection process ( $p$ $<0.001)$.Likewise, the physicians reported a higher cooperation level of the patients with the medical staff in the blood culture collection process in the general than in the geriatric hospital $(p<0.001)$. Regarding the question on the importance of the staff's assistance during the blood drawing process (in patient's arm fixation), there was a significant difference between the two kinds of hospitals, being graded significantly more important by the geriatric hospital doctors $(p=0.019)$. Additionally, there was a difference in the assessment of the overall importance of nursing staff's participation in blood culture collection being much higher in the geriatric than in the general hospital $(p=0.014)$. Likewise, ninety percent of the physicians from the geriatric hospital felt that a retraining program of blood culture collection techniques was important as opposed to $48 \%$ of those from the general hospital $(p<0.001)$.

\section{DISCUSSION}

Our ongoing educational program succeeded in reducing the rate of blood culture contaminations from $9 \%$ to $6 \%$. However this difference is not statistically significant $(p=0,21)$ and this rate is still higher than that reported by general hospitals and the rates often quoted in the medical literature ${ }^{15}$.

Qamruddin and his colleagues reported that an $8.8 \%$ rate of blood cultures contaminations dropped significantly when the protocol of blood culture sampling was carefully observed ${ }^{16}$. Bekeris and his colleagues ${ }^{10}$ and Eskira and his colleagues 
17 also reported decreased contamination rates by using devoted teams for blood culture collection.

Despite our improved techniques for aseptic blood culture sampling we did not reach the low contamination rate of general hospitals, and therefore, we presume that this discrepancy may be related to the differences between our patients and those of the general hospital. It is well known that many long-term care geriatric patients are characterized by low cognition and high level of disability (induced by paresis and spasticity). The process of blood culture sampling is complex and also requires the cooperation of the patients. Moreover, since cooperation is limited in most geriatric patients, this theoretically could be compensated by the active assistance of nursing staff members that could ensure the necessary sequential steps required: holding, immobilizing and sterilizing the venipuncture site, assuring sterile transfer of the blood sample to the culture bottle. A high contamination rate has a high economic impact estimated at about two million dollars per year as described in several studies ${ }^{6}$. Bates et al. found that contaminated results, compared with true-negative results, were independently associated with increased subsequent laboratory and intravenous antibiotic charges ${ }^{5}$. Souvenir et al. reported that almost half of the patients with the false-positive results were treated with antibiotics, often with Vancomycin ${ }^{17}$. According to their estimation, the additional costs associated with this unnecessary treatment were approximately 1,000 \$ per patient.

Reducing contamination rates would lead to improved specificity and better performance of this important test ${ }^{18}$. Factors that have been explored so far include skin preparation, culture bottle preparation, and single versus double needle for bottle inoculation, dedicated phlebotomy teams, and the use of commercial blood culture collection kits ${ }^{19}$. Part of these factors directly depended on the presence of trained staff that can ensure satisfactory blood culture collection techniques.

In conclusion, our study, the first undertaken in a geriatric hospital indicates that blood culture contamination rates are higher in geriatric hospitals and suggests that these could be reduced by a coordinated educational effort of the physicians combined with an increased number of nurse aids in the team that actually carries out the blood sampling process. Further reports from similar facilities are needed in order to support our conclusions which call for a reinforced team for the blood culture sampling in geriatric patients.
All authors have no financial foundation, and all authors have no conflict of interest.

\section{REFERENCES}

1. Lubart E, Segal R, Haimov E, Dan M, Baumoehl Y, Leibovitz A. Bacteremia in a Multilevel Geriatric Hospital. Journal of the American Medical Directors Association [Internet]. Elsevier BV; 2011 Mar;12(3):204-7. Available from: http://dx.doi.org/10.1016/i.jamda.2010.02.017

2. Bacteremia in a Multilevel Geriatric Hospital, Second Look 5 Years Later. British Journal of Medicine and Medical Research [Internet]. Sciencedomain International; 2013 Dec 21;4(8) 1729-38. Available from: http://dx.doi.org/10.9734/bjmmr/2014/6892

3. Weinstein MP. Blood Culture Contamination: Persisting Problems and Partial Progress. Journal of Clinical Microbiology [Internet]. American Society for Microbiology; 2003 Jun 1;41(6):2275-8. Available from: http://dx.doi.org/10.1128/jcm.41.6.2275$\underline{2278.2003}$

4. Richter SS, Beekmann SE, Croco JL, Diekema DJ, Koontz FP, Pfaller MA, et al. Minimizing the Workup of Blood Culture Contaminants: Implementation and Evaluation of a Laboratory-Based Algorithm. Journal of Clinical Microbiology [Internet]. American Society for Microbiology; 2002 Jul 1;40(7):2437-44. Available from: http://dx.doi.org/10.1128/jcm.40.7.2437$\underline{2444.2002}$

5. Bates DW. Contaminant blood cultures and resource utilization. The true consequences of false-positive results. JAMA: The Journal of the American Medical Association [Internet]. American Medical Association (AMA); 1991 Jan 16;265(3):365-9. Available from: http://dx.doi.org/10.1001/jama.265.3.365

6. Kim JY, Rosenberg ES. The Sum of the Parts Is Greater Than the Whole: Reducing Blood Culture Contamination. Ann Intern Med [Internet]. American College of Physicians; 2011 Feb 1;154(3):202. Available from: http://dx.doi.org/10.7326/0003-4819-154-3$\underline{\text { 201102010-00010 }}$

7. Weinstein MP, Towns ML, Quartey SM, Mirrett S, Reimer LG, Parmigiani G, et al. The Clinical Significance of Positive Blood Cultures in the 1990s: A Prospective Comprehensive Evaluation of the Microbiology, Epidemiology, and Outcome of Bacteremia and Fungemia in Adults. Clinical Infectious Diseases [Internet]. Oxford University Press (OUP); 1997 Apr 1;24(4):584-602. Available from: http://dx.doi.org/10.1093/clind/24.4.584 
8. Bates DW. Rapid classification of positive blood cultures. Prospective validation of a multivariate algorithm. JAMA: The Journal of the American Medical Association [Internet]. American Medical Association (AMA); 1992 Apr 8;267(14):1962-6. Available from: http://dx.doi.org/10.1001/jama.267.14.1962

9. Martin MA, Pfaller MA, Wenzel RP. Coagulasenegative staphylococcal bacteremia. Mortality and hospital stay. American Journal of Infection Control [Internet]. Elsevier BV; 1990 Feb;18(1):50. Available from: $\quad$ http://dx.doi.org/10.1016/0196$\underline{6553(90) 90211-a}$

10. Bekeris LG, Tworek JA, Walsh MK, Valenstein PN. Trends in blood culture contamination: a College of American Pathologists Q-Tracks study of 356 institutions. Arch Pathol Lab Med. 2005 Oct;129(10):1222-5. PubMed PMID: 16196507.

11. Youssef D, Shams W, Bailey B, O'Neil TJ, Al-Abbadi MA. Effective strategy for decreasing blood culture contamination rates: the experience of a veterans affairs medical centre. Journal of Hospital Infection [Internet]. Elsevier BV; 2012 Aug;81(4):288-91. Available from: http://dx.doi.org/10.1016/j.jhin.2012.05.014

12. Snyder SR, Favoretto AM, Baetz RA, Derzon JH, Madison BM, Mass D, et al. Effectiveness of practices to reduce blood culture contamination: A Laboratory Medicine Best Practices systematic review and meta-analysis. Clinical Biochemistry [Internet]. Elsevier BV; 2012 Sep;45(13-14):9991011. Available from: http://dx.doi.org/10.1016/j.clinbiochem.2012.06.0 $\underline{07}$

13. Ge $Y$, Liu $X Q, X u Y C$,et al. Blood collection procedures influence contamination rates in blood culture: a prospective study. Chin Med J (Engl). 2011; 124(23):4002-6.

14. Walther-Wenke G, Däubener W, Heiden $M$, Hoch J, Hornei B, Volkers P, et al. Effect of Safety Measures on Bacterial Contamination Rates of Blood Components in Germany. Transfusion Medicine and Hemotherapy [Internet]. S. Karger AG; 2011;38(4):231-5. Available from: http://dx.doi.org/10.1159/000330417

15. Blood culture contamination: a College of American Pathologists Q-Probes study involving 640 institutions and 497134 specimens from adult patients. Arch Pathol Lab Med. 1998; 122(3):21621.

16. Qamruddin A, Khanna N, Orr D. Peripheral blood culture contamination in adults and venepuncture technique: prospective cohort study. Journal of Clinical Pathology [Internet]. BMJ; 2008 Apr
1;61(4):509-13. Available from: http://dx.doi.org/10.1136/icp.2007.047647

17. Eskira S, Gilad J, Schlaeffer P, Hyam E, Peled N, Karakis I, et al. Reduction of blood culture contamination rate by an educational intervention. Clinical Microbiology and Infection [Internet]. Elsevier BV; 2006 Aug;12(8):818-21. Available from: http://dx.doi.org/10.1111/j.1469$\underline{0691.2006 .01446 . x}$

18. 18. Souvenir D.,Anderson DE., Palpant JS et al. Blood cultures positive for coagulase-negative staphylococci: antisepsis, pseudobacteremia and therapy of patients. J.Clin.Microbiol. 1998; 36:1923-1926.

19. Hall KK, Lyman JA. Updated Review of Blood Culture Contamination. Clinical Microbiology Reviews [Internet]. American Society for Microbiology; 2006 Oct 1;19(4):788-802. Available from: http://dx.doi.org/10.1128/cmr.00062-05 